\title{
Pensar-com Tecnologias Digitais de Matemática Dinâmica
}

\author{
Marcus Basso - IME - PPGEMat - PGIE - UFRGS \\ mbasso@ufrgs.br \\ Márcia Rodrigues Notare - IME - PPGEMat - PGIE - UFRGS - \\ marcia.notare@gmail.com
}

\begin{abstract}
Resumo
Nesse artigo analisa-se o papel do uso dos recursos digitais de matemática dinâmica na construção de conceitos a partir da resolução de situações-problemas propostos a um grupo de estudantes de curso de Informática na Educação e o pensar em matemática. De caráter exploratório, com base na Teoria Construcionista de Papert, são analisadas possíveis contribuições que o uso de artefatos digitais oferecem na construção de conceitos matemáticos. A pesquisa aponta para a necessidade de, na construção de situações-problemas, considerar questões nas quais a tecnologia torna-se um instrumento na mobilização do pensamento matemático.
\end{abstract}

Palavras-chave: matemática dinâmica, tecnologias digitais, educação matemática

\section{Think-with Digital Technologies of Dynamic Mathematics}

\begin{abstract}
This article analyzes the role of the use of digital resources of dynamic mathematics in building concepts from the proposed resolution of problem situations to a group of students of informatics in education and thinking in mathematics. This exploratory study, based on construcionism theory of Papert are analyzed potential contributions that the use of digital artefacts in the construction of mathematical concepts. The research points to the need, in the construction of situations-problems, consider matters in which the technology becomes an instrument in the mobilization of mathematical thinking.
\end{abstract}

Key-words: dynamic mathematics, digital technology, mathematics education

\section{Introdução - contexto do problema}

Estudos abordando o impacto do uso de recursos digitais que oferecem a possibilidade de operar com matemática estão em uma curva ascendente na literatura da Informática na Educação e na Educação Matemática. Um levantamento das publicações tratando de Matemática na Revista Brasileira de Informática na Educação (RBIE, 2015) de 1999 a 2014, permite identificar 17 trabalhos publicados de 1999 a 2011 e 19 trabalhos de 2012 a 2014, evidenciando o crescimento nas produções e o interesse nessa temática. Mais recentemente, o aspecto do uso de recursos de matemática dinâmica se tornou um tema em evidência nas pesquisas em Tecnologias Digitais na Educação Matemática. A programação do XI Encontro Nacional de Educação Matemática (XI ENEM, 2015) contemplou 40 trabalhos, incluindo minicursos, comunicações científicas e relatos de experiência, sobre o tema. De forma similar, essa situação também se reflete nas publicações da Revista de Novas Tecnologias na Educação (RENOTE, 2015). De 2003 a 2015, 183 artigos sobre tecnologias e matemática foram publicados, e destes, cerca de $10 \%$ investigam ou relatam experiências com o uso de matemática dinâmica. 
Assim, o presente artigo se insere no conjunto de produções voltadas para compreender o impacto do uso de recursos das tecnologias digitais de informação e comunicação nos processos envolvendo a apropriação de tecnologias digitais e o conhecimento matemático. Em particular, discute-se nesse trabalho o uso de recursos digitais de matemática dinâmica no fazer matemática propostos a um grupo de estudantes em Seminário do Curso de Pós-graduação em Informática na Educação da Universidade Federal do Rio Grande do Sul (UFRGS). O artigo está centrado na análise de situações-problemas com potencial para desencadear o pensamento matemático via matemática dinâmica (MD).

Inicialmente, uma questão se impõe: o que caracteriza a matemática dinâmica?

De acordo com Moreno-Armella, Hegedus e Kaput, (2008, p.103), "A natureza dos símbolos matemáticos têm evoluído nos últimos anos de um caráter estático, inerte, para objetos dinâmicos ou diagramas que são construtíveis, manipuláveis e interativos".

Consideremos o exemplo de trabalhar com a função $\mathbf{y}=\mathbf{a x}^{2}+\mathbf{b x}+\mathbf{c}$, de coeficientes $\mathbf{a}, \mathbf{b}$ e $\mathbf{c}$ reais e as modificações na representação gráfica dessa relação funcional provocadas por alterações nesses coeficientes. Não há dúvida que muitos operaram com esses conceitos e objetos matemáticos a partir de representações estáticas, sejam aquelas registradas pelo professor com quadro-giz ou no caderno com papel-lápis. Um sem número de gráficos e textos foram e, ainda hoje, são representados usando tais recursos. Para cada alteração em apenas um dos coeficientes, uma nova representação gráfica e algébrica no sistema quadro-giz (ou caderno-lápis) deve ser feita. Essa mesma situação, agora representada com, por exemplo, o software GeoGebra, oferece a possibilidade de manipular os coeficientes e observar de forma dinâmica, ou seja, com movimento, as alterações gráficas provocadas. Ademais, é possível observar estas alterações tanto do ponto de vista geométrico quanto do ponto de vista algébrico. Agrega-se a essa situação, o aspecto do próprio aprendiz poder manipular, controlar, observar, agir sobre o objeto digital, caracterizando uma situação ativa no processo de pensar na matemática envolvida.

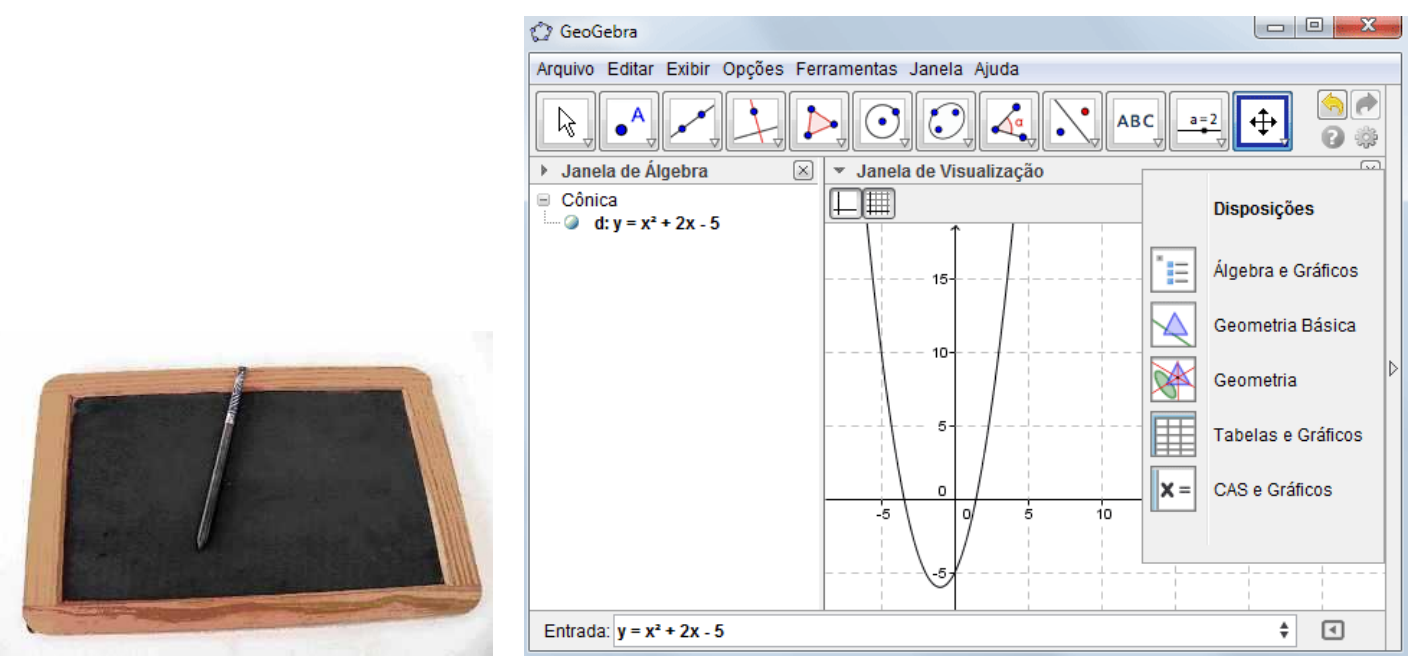

Figura 1: quadro-giz, cursor-tela - estático versus dinâmico

Assim, analisar situações nas quais se utilizam essas ferramentas para o pensamento (Papert, 1993) ou, como citado por Gravina e Basso (2012, p. 13),

"Nessa direção temos a provocativa expressão de Shaffer e Clinton (2006) "ferramentaparapensamentos (toolforthougths)" - cunhada com o propósito de registrar uma visão que considera que sujeitos e artefatos tecnológicos podem se colocar em 
situação de simbiose, em processo mútuo de ação e reação. Ou seja, o artefato também tem o poder de agir sobre o sujeito, daí a expressão que funde as duas palavras."

é o aspecto central do presente trabalho. Estamos particularmente interessados em analisar situações nas quais o uso dos recursos digitais coloca o aprendiz em uma posição que favoreça a construção de conceitos e o pensar em matemática. Para isso, elencamos duas situações-problemas propostas em disciplina do Doutorado em Informática na Educação da UFRGS.

Tendo como foco estudar processos de gênese instrumental pessoal e profissional (Haspekian, 2013, Lagrange, 2013) no uso de tecnologias digitais a partir da utilização de tecnologias digitais no ensino e aprendizagem de matemática, nessa disciplina os estudantes são convidados a resolver e refletir sobre problemas de geometria usando, dentre outros softwares, o GeoGebra. Frente a estas situaçõesproblemas, analisamos possibilidades oferecidas por elas nos processos de pensar e fazer matemática utilizando o GeoGebra como recurso digital de matemática dinâmica.

$\mathrm{Na}$ sequência do artigo, apresentamos a fundamentação teórica do trabalho e duas situações-problemas acompanhadas de análises de seu potencial de uso para desencadear novas formas de pensar em matemática. Finalizamos apresentando resultados e perspectivas de continuidade de estudos na direção sugerida neste artigo.

\section{Fundamentos teóricos}

O surgimento das tecnologias e dos ambientes dinâmicos proporcionou a evolução tanto da Matemática, quanto da Educação Matemática. Em Matemática, os computadores fomentaram a descoberta de novos campos. Na Educação Matemática, eles têm tornado mais acessíveis alguns problemas e ideias, proporcionado novas formas de representar e manipular os objetos matemáticos.

Pea (1987) investigou sobre o que de novo podemos fazer com os computadores, que até então não podíamos? O que a tecnologia tornou possível em termos de pensamento matemático? Para Pea (1987), os computadores afetam as pessoas e suas formas de pensar: o que as crianças conseguiam fazer em termos de matemática em determinada idade ou estágio do desenvolvimento cognitivo até então estava restrito ao meio estático do lápis e papel. Hoje, com os recursos tecnológicos interativos e dinâmicos, temos um ganho de compreensão, proporcionado pelas representações agora acessíveis por meio desses ambientes. Os recursos tecnológicos e a possibilidade de representação e manipulação de objetos matemáticos abrem novas possibilidades para o pensamento matemático.

Não estamos falando aqui em utilizar a tecnologia para proporcionar mais praticidade e rapidez na execução de algoritmos ou na resolução de problemas, nem mesmo no uso da tecnologia para dar praticidade ao trabalho do professor ou para tornar a aula mais atraente e interessante para o aluno. Estamos falando em utilizar a tecnologia de modo a desencadear o pensamento matemático, a proporcionar aos alunos possibilidades para acessar e manipular objetos matemáticos até então não acessíveis.

Para Goldenberg (2000), a tecnologia pode ajudar os alunos a desenvolver novas formas de olhar para os problemas matemáticos, ajudando-os a construir modelos mentais e a desenvolver habilidades de generalização e flexibilidade de pensamento. Os alunos que experimentam situações nas quais é possível movimentar e modificar objetos geométricos na tela do computador, começam a aprender como realizar os mesmos tipos de experiências em suas mentes, na ausência do recurso tecnológico.

Moreno-Armella (2004) defende que as tecnologias influenciam na forma de pensar em Matemática. De acordo com Balacheff e Kaput (1996), o principal impacto 
da tecnologia na Educação Matemática é de ordem epistemológica e cognitiva, porque ela contribui para a produção de uma nova forma de realismo dos objetos matemáticos. As versões virtuais de objetos matemáticos produzem a sensação de existência material, dada a possibilidade de alterá-los na tela do computador. Nessa perspectiva, os recursos computacionais podem ser gradualmente incorporados à atividade dos alunos, transformando-se em verdadeiros instrumentos matemáticos, que possibilitam pensar em matemática de uma nova maneira.

Dessa forma, torna-se cada vez mais importante refletir sobre o papel da tecnologia na Educação Matemática: a tecnologia deve ser usada para resolver um problema ou para ajudar os alunos a pensar sobre um problema, analisar um processo, gerar uma prova? Para determinado conteúdo ou conceito de matemática, o papel da tecnologia é substituir uma capacidade que o aluno poderia desenvolver ou desenvolver a capacidade do aluno de pensar?

Para tirar proveito das potencialidades da tecnologia no desenvolvimento do pensamento matemático, é preciso considerar que não basta fazer uma boa escolha do recurso tecnológico. Os problemas que são propostos com estes recursos é que podem fazer diferença. Assim, com a tecnologia, o que, de fato, deve mudar, é a escolha do conjunto de problemas e a forma como eles serão apresentados aos alunos. Alguns problemas são difíceis de serem abordados apenas com lápis e papel. Alguns problemas, por exemplo, exigem dos alunos a realização de experiências com objetos matemáticos, para possibilitar a observação de seus comportamentos diante da manipulação de seus elementos. Nas séries iniciais, objetos físicos manipulativos podem fornecer este suporte visual e experimental para as crianças. Eles servem como modelos físicos temporários para desencadear ideias matemáticas, ou seja, constituem objetos nos quais as crianças podem ver com os olhos e manipular com as mãos, enquanto aprendem a ver e manipular as ideias matemáticas em suas mentes (Goldenberg, 2000). Em níveis mais elevados, muitas ideias matemáticas não têm suporte nesses modelos físicos. Nestes casos, os computadores podem fornecer a possibilidade de manipulação virtual, em situações nas quais dispositivos físicos não existem ou não são acessíveis.

É importante, no momento de pensar em atividades com o uso de tecnologias para a sala de aula, ter claro os objetivos que queremos alcançar e escolher a tecnologia de modo a atendê-los, ao invés de simplesmente utilizar a tecnologia para tornar a aula mais atraente, mas de forma tangente e superficial, ou até mesmo prejudicial.

Para Pea (1987), as atividades propostas em ambientes computacionais devem ser pensadas de modo a promover o pensamento matemático, e não para facilitar os procedimentos de resolução dos problemas. Entendemos que um bom uso dos recursos tecnológicos deve proporcionar situações que ajudem os alunos a pensar matematicamente, fornecendo atividades de matemática cujos objetivos vão além do "aprender matemática", em direção ao "fazer matemática". Pensar em problemas amplos, em que a matemática a ser aprendida é essencial para lidar com a situação deve ser o foco. A matemática torna-se funcional, uma vez que as tecnologias levam ao desenvolvimento do pensamento matemático como forma de resolver um problema $\mathrm{e}$ não como um fim em si mesmo.

Nessa perspectiva, o uso das tecnologias na Educação Matemática deve considerá-la como uma ferramenta que leve o aluno a compreender que pode se tornar um sujeito capaz de criar e pensar em Matemática.

A seguir, discutimos especificamente os ambientes de geometria dinâmica e seu papel no desenvolvimento do pensamento geométrico. 


\subsection{A Geometria Dinâmica e o Pensamento Geométrico}

Os ambientes de geometria dinâmica (GD), um caso particular de matemática dinâmica, são excelentes micromundos para expressar ideias matemáticas (Balacheff e Kaput, 1996). Em ambientes de GD, é possível alcançar um nível elevado de realismo para representar diferentes objetos matemáticos, pois oferecem a possibilidade de manipulação direta de construções geométricas, que permitem visualizar conceitos de geometria a partir do estudo de propriedades invariantes dessas construções enquanto seus componentes são movimentados na tela. A afirmação de uma propriedade geométrica torna-se, neste novo campo de experimentação, a descrição de um fenômeno geométrico acessível à observação. A tecnologia leva os alunos em direção a um amplo espaço de experiências. Dessa forma, o ambiente de GD constitui-se como um espaço em que os alunos podem tornar possíveis suas ideias informais, para dar início a um processo de coordenação com ideias mais formalizadas sobre determinado assunto.

A exploração de figuras e de suas propriedades dá origem ao reconhecimento de um sistema de relações geométricas, que em última análise constituem o objeto geométrico. É importante compreender o papel epistemológico das ferramentas tecnológicas quando estão articuladas com o pensamento matemático, ou seja, compreender que conhecimento matemático emerge a partir das interações com essas ferramentas.

Nesses ambientes, os alunos precisam decidir se eles construíram o que pretendiam construir inicialmente. O critério para essa decisão é baseado na evidência perceptiva, ou seja, a construção deve manter suas propriedades e relações internas quando seus componentes básicos são movimentados na tela.

Para que os ambientes de GD tenham papel importante na aprendizagem de geometria, é preciso ter cuidado na escolha das situações propostas aos alunos e cuidado na exploração do fenômeno da tela do computador. Um problema clássico de geometria pode se tornar obsoleto em um ambiente de GD, e por outro lado, esses ambientes abrem possibilidades para explorar problemas que estão fora do alcance do lápis e papel.

Uma das principais contribuições dos ambientes de geometria dinâmica é a evolução do conceito de prova em geometria. Com estes ambientes, professores podem ser encorajados a oferecer atividades que favoreçam a exploração, a conjectura, a argumentação, a discussão e, finalmente, a prova. Neste sentido, a noção de prova em matemática escolar muda com o surgimento da GD, pois estes ambientes possibilitam de forma surpreendente a experimentação (Sinclair e Robutti, 2013). Hoje, quando falamos em prova, podemos entendê-la como um "processo de provar", no qual procurase pelos argumentos que explicam a veracidade de uma afirmação, de acordo com regras de consequência lógica.

O processo de prova consiste em duas fases: a formulação de uma conjectura e a construção de uma prova. Na primeira fase, o sujeito explora a situação, formula uma conjectura e pesquisa por elementos que serão organizados mais tarde. Na segunda fase, esses elementos devem ser colocados em ordem de acordo com as regras da consequência lógica. Como destacamos acima, a mudança de foco para o "processo de provar" é especialmente relevante em ambientes de GD, no sentido de que a primeira fase do processo difere radicalmente dos ambientes de papel e lápis, que, por sua vez, afeta a evolução da segunda fase, ou seja, da construção da prova propriamente dita.

Figuras dinâmicas podem ter um importante papel no processo de prova, no sentido de mediar a exploração e realização de conjecturas (primeira fase) e as propriedades que devem ser identificadas para, em seguida, organizar logicamente as ideias (segunda fase). Neste sentido, a figura dinâmica construída em um ambiente de 
GD e os esquemas de uso ativados nesse processo pelos alunos podem ser considerados mediadores entre as primeira e segunda fases, proporcionando assim continuidade, ou seja, entre uma conjectura (declaração hipotética) e a prova correspondente (sequência lógica dedutiva de declarações). A internalização dos esquemas de uso do artefato e sua transformação em um instrumento pode ajudá-los na construção da prova como produto final. (Rabardel, 1995)

Nessa perspectiva, um ambiente de GD pode ser visto como um artefato nas mãos do usuário. Esse artefato pode ser transformado em instrumento pelo usuário, de acordo com os esquemas de uso que são ativados ao explorar determinada tarefa. Vários estudos têm documentado como esse processo ocorre em ambientes de GD. Se os estudantes internalizam o uso de um ambiente de GD, o artefato torna-se um meio de mediação semiótica (Bussi e Mariotti, 2008) que dá origem à resolução do problema em questão.

A ação de arrastar (característica central dos ambientes de GD) muda o aspecto figural de uma construção geométrica, mas não o aspecto conceitual, uma vez que todas as propriedades do objeto geométrico são mantidas. Esta dualidade figural/conceitual não é possível em um ambiente estático de lápis e papel, uma vez que os aspectos figurais são tratados em um registro visual e um conceito é tratado em um registro discursivo. As provas geométricas são concentradas em objetos teóricos, e não apenas em desenhos específicos. Assim, o papel que o recurso de movimento pode desempenhar na articulação entre os aspectos figural e conceitual é particularmente importante, pois proporciona acesso ao mundo da teoria geométrica.

A ação de arrastar pode ser agrupada em duas categorias mais amplas: arrastar para explorar e conjecturar e arrastar para a validar uma conjectura ou prova. No primeiro caso, os alunos arrastam objetos geométricos para observar a figura em busca de regularidades e invariantes. Uma vez que as encontraram, eles a expressam por meio de uma conjectura, avançando do campo gráfico para o campo teórico. Por outro lado, os alunos podem arrastar um objeto geométrico para verificar uma conjectura já descoberta.

Outro recurso importante dos ambientes de GD é o recurso de rastro. Este recurso tem potencial para fornecer evidências exploratórias em determinados problemas, dando suporte para a investigação sobre uma característica específica da situação apresentada ou para o estabelecimento de relações entre seus componentes.

\section{Matemática dinâmica na resolução de situações-problemas}

Nessa seção apresentamos duas situações-problemas nas quais os estudantes deveriam utilizar o software de MD GeoGebra para resolvê-las. Cada situação-problema será acompanhada de resoluções propostas pelos estudantes bem como das análises correspondentes.

As duas situações-problemas foram selecionadas para ilustrar o potencial dos ambientes de MD para promover o pensar em matemática, nas quais a tecnologia tornase um instrumento matemático importante para tornar possível a solução do problema. A característica principal que difere os dois problemas está centrada na ação de arrastar proporcionada pela MD. Na primeira situação-problema, veremos o recurso de arrastar sendo usado pelos alunos para testar e validar uma construção já realizada, observando se as propriedades impostas pelo problema são mantidas sob ação do movimento. Na segunda situação-problema, o recurso de arrastar é usado para explorar e realizar uma conjectura. Em ambos os casos, temos a tecnologia como promotora do pensamento geométrico e crucial para a solução do problema. 
A situação-problema 1 tem o seguinte enunciado:

Construa as figuras abaixo, a partir do círculo maior e de tal forma que, ao movimentarmos o ponto $P$ da construção, todos os círculos se mantenham "encaixados".

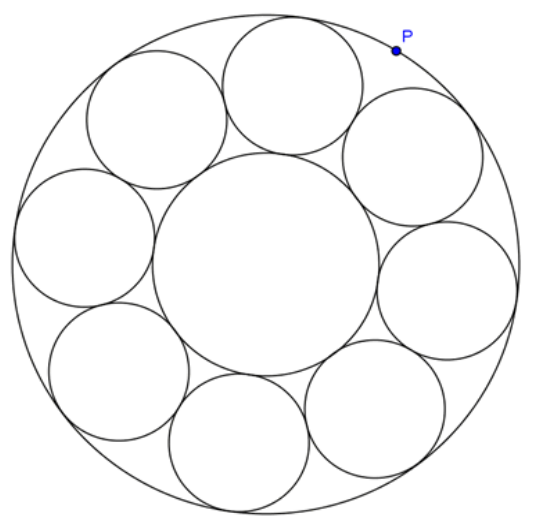

Nesta situação-problema, os alunos devem elaborar uma estratégia para a construção da figura, a partir de propriedades geométricas, de modo que os círculos permaneçam encaixados sob ação do movimento. O problema está em encontrar, geometricamente, centro e raio de um dos círculos (os demais podem ser obtidos pela rotação do primeiro em torno do centro do círculo maior). Podemos perceber que os círculos menores possuem três pontos de tangência importantes para a contrução: dois pontos de tangências com círculos de mesmo raio e um ponto de tangência com o círculo maior. Dessa forma, é possível conjecturar que o círculo a ser construído pode ser tangente aos três lados de um triângulo. Neste momento, propriedades geométricas devem ser utilizadas para construir os lados do triângulo e o círculo procurado, em especial, o incentro do triângulo (ponto de intersecção das bissetrizes internas do triângulo), que determina o centro da circunferência inscrita no mesmo. A Figura 2 ilustra a construção do problema.

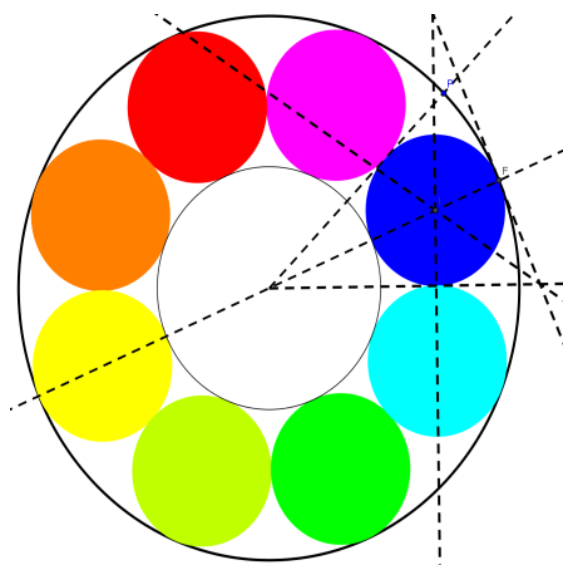

Figura 2: Construção dinâmica da situação-problema

No caso desse problema, os alunos utilizam o recurso de movimento proporcionado pela MD para validar a construção realizada, ou seja, verificar se os círculos permanecem tangentes e encaixados, como sugere o problema. Sem o dinamismo proporcionado pela $\mathrm{MD}$, o momento de conjecturas e refutações não seria possível, enfraquecendo o problema. 
A situação-problema 2 tem o seguinte enunciado:

Considere uma circunferência de centro $O$ e um ponto $P$ em seu interior. Determine o lugar geométrico dos centros das circunferências que passam por $P$ e são tangentes à circunferência de centro $O$.

Percebe-se que a resolução desse problema envolve duas fases. Na primeira fase, o aluno precisa explorar a situação apresentada para formular uma conjectura. Esta fase de exploração permite que o aluno busque por elementos, propriedades, regularidades que indiquem um caminho para a solução do problema. Os fatos observados na primeira fase, devem ser organizados logicamente na fase seguinte, na qual o aluno deve buscar argumentos matemáticos que justifiquem e provem a conjectura inicial, ou a refutem.

É possível notar que a solução deste problema em ambiente de MD possibilita um rico espaço para a exploração, na qual os alunos podem realizar a construção da situação, conforme ilustra a Figura 3a, a partir de propriedades geométricas, e utilizar os recursos de movimento e rastro para conjecturar a solução.

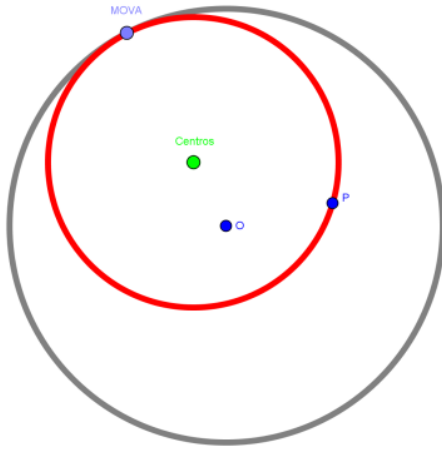

a

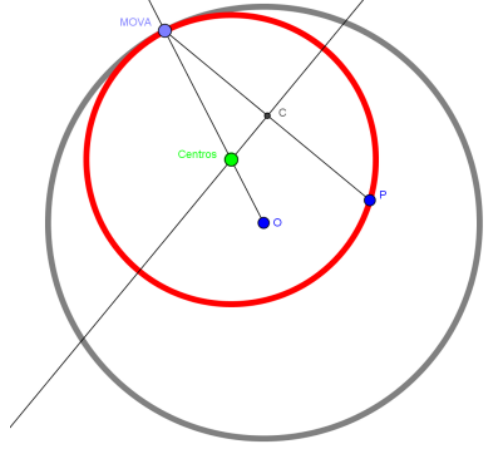

b

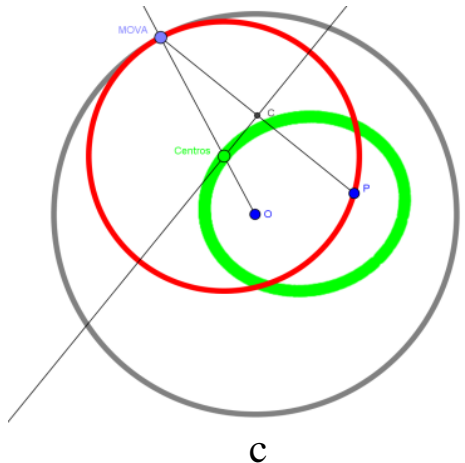

c

Figura 3: Figura que representa a situação-problema

O primeiro desafio que se impõe neste problema é realizar uma construção que satisfaça as condições do problema e mantenha-se estável ao ser movimentada, ou seja, a circunferência interna vermelha deve permanecer tangente à circunferência externa dada e sempre passar pelo ponto P. Para realizar a construção, é preciso encontrar o centro da circunferência vermelha. E para isso, alguns conceitos e propriedades geométricas devem ser considerados: (1) a circunferência vermelha deve passar pelo ponto $\mathrm{P}$ dado pelo enunciado do problema e pelo ponto de tangência com a circunferência dada, ou seja, são conhecidos dois pontos que pertencem à circunferência; (2) a mediatriz de qualquer corda de uma circunferência passa pelo seu centro; (3) os centros das duas circunferências e o ponto de tangência entre ela estão alinhados. Considerando estas propriedades, o centro procurado é dado pelo ponto de intersecção entre a reta mediatriz da corda e a reta que contém os centros e o ponto de tangência, como ilustra a Figura $3 b$.

Uma vez construída a figura dinâmica, é possível habilitar o recurso de rastro do centro da circunferência vermelha para, a partir do movimento, observar o lugar geométrico procurado. Na Figura 3c, é possível conjecturar que o lugar geométrico dos centros das circunferências que passam por $\mathrm{P}$ e são tangentes à circunferência de centro O é uma elipse com focos nos pontos $\mathrm{O}$ e P. É importante ressaltar que a utilização do ambiente de MD para explorar a situação torna possível a representação e manipulação do objeto matemático, que em ambiente de lápis e papel seria dispendioso e pouco preciso. Conforme defendido por Balacheff e Kaput (1996), temos a tecnologia, em 
especial a GD, tornando possível uma nova forma de realismo dos objetos matemáticos e produzindo a sensação de existência material, dada a possibilidade de alterá-los na tela do computador. Os alunos que incorporam esses recursos para resolver problemas matemáticos, têm em mãos verdadeiros instrumentos matemáticos, que possibilitam pensar em matemática de uma nova maneira.

Contudo, o problema não termina aqui. Agora, é o momento de dar início ao processo de prova: porque, de fato, o lugar geométrico procurado é uma elipse? Quais argumentos justificam e provam esta conjectura?

Esse constitui o passo seguinte no processo de fazer matemática pensando-com o instrumento de MD.

\section{Considerações finais}

Pensar-com. Essa expressão, simples na forma mas potente no seu conteúdo constitui o pilar desse artigo. Tal ideia pode estabelecer uma base conceitual para criar situações-problemas que, demandando o uso de recursos digitais, favoreça o uso e o pensar em matemática.

Argumentar que as tecnologias digitais devem ser utilizadas em classes de matemática porque os estudantes têm acesso a esses recursos oferece apenas uma perspectiva para se examinar a introdução desses recursos na Escola. Pretendemos ir além dessa argumentação, pensando nas decisões a serem tomadas quanto ao potencial da tecnologia no fazer matemática dos alunos. Não basta ter acesso, embora necessário, a ambientes digitais. O universo de objetos e de propostas é que podem conferir a esses ambientes a potencialidade para despertar modos criativos de resolver problemas em e com matemática.

\section{Referências}

BALACHEFF, N; KAPUT, J. Computer-Based Learning Environments in Mathematics. In: BISHOP, A.; CLEMENTS, K.; KEITEL, C.; KILPATRICK, J.; LABORDE, C. (Eds), International Handbook of Mathematics Education. Springer, 1996, p. 469-500.

GOLDENBERG, P. Thinking (And Talking) About Technology in Math Classrooms. In: Education Development Center, 2000. Disponível em: http://www2.edc.org/mcc/PDF/iss_tech.pdf

GRAVINA, M. A., BASSO, M. V. A., Mídias Digitais na Educação Matemática. In: GRAVINA, M. A., BÚRIGO, E. Z., BASSO, M.V.A., GARCIA, V.C.V. (org.) Matemática, Mídias Digitais e Didática - tripé para formação do professor de Matemática. Porto Alegre: Evangraf, 2012. Disponível em: http://www.ufrgs.br/espmat/livros/livro2-matematica_midiasdigitais_didatica.pdf. Acesso em 30 out 2015.

HASPEKIAN, M. Enseigner avec un tableur: quelles genèses instrumentales? In: Les technologies numériques pour lénseignement:usages, dispositifs et genèses. Toulouse: OCTARÈS Editions, 2013.

LAGRANGE, J.B. Les technologies numériques pour lénseignement: usages, dispositifs et genèses. Toulouse: OCTARÈS Editions, 2013. 
BUSSI. M. G. B., MARIOTTI, M. A. Semiotic mediation in the mathematics classroom: artifacts and signs after a Vygotskian perspective. In: ENGLISH, L., BUSSI, M.G.B., JONES, G., LESH, R., TIROSH, D. (Eds.), Handbook of International Research in Mathematics Education (pp. 746-783). Mahwah, NJ: Lawrence Erlbaum, 2008.

MORENO-ARMELLA, L. Mathematical thinking and technology: some views on their co-evolution. In: TSG15-ICME-10, Copenhagen. Disponível em http://matrix.skku.ac.kr/sglee/album/TSG 15_The role and use of technology in the teaching and learning of mathematics.pdf. Acesso em 21 out 2015.

MORENO-ARMELLA, L. HEGEDUS, S. J., KAPUT., J.J. From static to dynamic mathematics: historical and representational perspectives. Educational Studies in Mathematics. v.68, p 99-111, 2008.

OLIVE, J., MAKAR, K., HOYOS, V., KOR, L. K., KOSHELEVA, O., STRÄßER, R. Mathematical Knowledge and Practices Resulting from Access to Digital Technologies. In: HOYLES, C., LAGRANGE, J. B. (Eds.) Mathematics Education and Technology-Rethinking the Terrain. The 17th ICMI Study. Londres: Springer, 2010 .

PAPERT, S. A máquina das crianças: repensando a escola na era da informática. Porto Alegre: Artmed, 2008.

PEA, R. (1987). Cognitive technologies for mathematics education. In A.H. Schoenfeld (Ed.),Cognitive Science and Mathematics Education (pp. 89-122). Hillsdale: Lawrence Erlbaum. http://web.stanford.edu/ roypea/RoyPDF\%20folder/A41_Pea_87b.pdf

RABARDEL, P. Les hommes et les technologies: une approche cognitive des instruments contemporains. Paris: Armand Colin, 1995.

RBIE. Revista Brasileira de Informática na Educação. Comissão Especial de Informática na Educação da Sociedade Brasileira de Computação. 2015. Disponível em: http://www.br-ie.org/rbie. Acesso em 20 out 2015.

RENOTE. Revista Novas Tecnologias na Educação. Centro de Novas tecnologias na Educação. Universidade Federal do Rio Grande do Sul, Porto alegre, 2015. Disponível em: http://seer.ufrgs.br/index.php/renote/index. Acesso em 20 out 2015.

SACRISTÁN, A. I., CALDER, N., ROJANO, T., TRIGO, M.S., FRIEDLANDER, A., MEISSNER, H., TABACH, M., MORENO, L., PERRUSQUIA, E. The Influence and Shaping of Digital Technologies on the Learning - and Learning Trajectories - of Mathematical Concepts. In: HOYLES, C., LAGRANGE, J. B. (Eds.) Mathematics Education and Technology-Rethinking the Terrain. The 17th ICMI Study. Londres: Springer, 2010.

SINCLAIR, N and ROBUTTI, O. Technology and the Role of Proof: The Case of Dynamic Geometry. In: CLEMENTS, M.A., BISHOP, A., KEITEL, C, KILPATRICK, J., LEUNG, F. (Eds.). Third International Handbook of Mathematics Education, New York: Springer, 2013, p. 571-596. 